\title{
Cephalometric Assessment of Dentoskeletal Characteristics in Children with Digit-sucking Habit
}

\author{
Tenali Sushmitha Singh ${ }^{1}$, Enuganti Sridevi ${ }^{2}$, Avula Jogendra Sai Sankar ${ }^{3}$, Pranitha Kakarla ${ }^{4}$, Siva Sai Krishna Vallabaneni ${ }^{5}$,
} Mukthineni Sridhar ${ }^{6}$

\begin{abstract}
Background: Nonnutritive sucking can turn into a continuous behavior practiced unconsciously, leading to a deleterious oral habit. Digit-sucking habits are an important etiological factor for malocclusion.

Aim: To investigate the effect of the digit-sucking habit on dentofacial structures by employing a cephalometric analysis.

Materials and methods: Selected 120 children were grouped as 60 with the digit-sucking habit and 60 without the digit-sucking habit in the age ranging between 6 and 12 years without gender discrimination. All were subjected to the standardized cephalometric technique, radiographs were traced by a single operator on a standard matte acetate tracing paper in a darkened room, and a total of 8 linear and 11 angular variables were measured for each patient in both the groups.

Statistical analysis: The unpaired Student's $t$-test was used to compare the mean difference between the two groups.

Results: The digit-sucking group showed significant difference in linear skeletal measurements such as value from anterior nasal spine (ANS) to posterior nasal spine (PNS), condylon to gnathion, nasion to ANS, sella to basion, and angular measurements such as angle between maxillary incisor to cranial plane, mandibular incisors to mandibular plane, sella nasion to point A, sella nasion to point B, and mandibular plane to cranial plane, when compared to the control group.

Conclusion: Within the confined parameters, digit sucking has led to significant variations in certain dental and skeletal cephalometric measurements.

Keywords: Digit sucking, Lateral cephalometry, Malocclusion.

International Journal of Clinical Pediatric Dentistry (2020): 10.5005/jp-journals-10005-1761
\end{abstract}

\section{INTRODUCTION}

Most of the valuable contributions to our knowledge of orofacial behavior have been made by astute clinicians who assert that the form of oral structures determines their function. ${ }^{1}$ Nonetheless, the myofunctional hypothesis proclaim that teeth move to occupy a neutral zone and any change in muscle force equilibrium can initiate morphological variation in the normal configuration of the teeth and the supporting bone, or it can enhance an already existing inherent malocclusion due to compensatory or adaptive muscle activity and functions. ${ }^{2}$

Many activities do not normally enter consciousness; movements follow a stimulus as a patterned behavior. ${ }^{1}$ In children, oral habits have concerned dentists and other healthcare professionals for many years because of the harmful unbalanced pressures, which may be brought to bear upon the immature, highly malleable alveolar ridges, the potential changes in the position of the teeth, and occlusion that may become abnormal if habits are continued beyond preschool age. ${ }^{3}$

One of the most common and earliest repetitive behaviors seen in the infantile period is digit sucking with prevalence between $61 \%$ and $90 \%$. It may extend until the period of $1-4$ years, 6 years, and $7-11$ years with the incidence of 46,13 , and $6 \%$, respectively. ${ }^{4}$ It has a well-established reputation within the orthodontic profession for causing anterior open bites, an increased overjet, high palatal vault, abnormal facial growth, posterior crossbites, crowding, increased probability of developing class II malocclusion, and further disturbs the normal development of the orofacial system causing facial deformities. The literature evidenced that these changes cause discernible disfigurements if persist beyond preschool age, ensuing
${ }^{1-6}$ Department of Pedodontics, Sibar Institute of Dental Sciences, Guntur, Andhra Pradesh, India

Corresponding Author: Avula Jogendra Sai Sankar, Department of Pedodontics, Sibar Institute of Dental Sciences, Guntur, Andhra Pradesh, India, Phone: +91 9346550646, e-mail: saisamata@gmail.com How to cite this article: Singh TS, Sridevi E, Sai Sankar AJ, et al. Cephalometric Assessment of Dentoskeletal Characteristics in Children with Digit-sucking Habit. Int J Clin Pediatr Dent 2020;13(3): 221-224.

Source of support: Nil

Conflict of interest: None

complicated surgical orthognathic procedures, multibanded mechanotherapy at a later age. ${ }^{5}$

Hence, one of the most valuable services that can be rendered as part of an interceptive orthodontic procedure is intervening oral habits during the primary and mixed dentition stages, which can enable the greatest possible control over growth changes and occlusal development. A pedodontist is at an advantage that he/she can see the child during the period that the habit is developing and gets the opportunity to examine the child before the detrimental effects of the habit manifest.

As the emerging field of myofunctional therapy spreads its wings, it is important to revisit the foundation that this field is built upon. With the advent of unique hybrid developments such as myobrace and preorthodontic trainer systems, this field has enjoyed great popularity and proven to elicit impressive results for habit correction and proper retraining of the oral musculature. It would 
be beneficial if we can exactly know where the skeletal changes have occurred secondary to the habit for proposing more accurate decisions on the time and type of intervention.

Thus, the present cephalometric study was aimed to evaluate the effect of the digit-sucking habit on the dentofacial skeleton by assessing the linear and angular measurements of various dental and skeletal changes.

\section{Materials and Methods}

Ethical clearance from the institutional ethical committee and an informed written consent from parents/guardians of the selected children were obtained prior to the conduct of this cephalometric study.

\section{Selection of the Cases}

Healthy patients in the age range of 6-12 years with and without the history of digit sucking were included in the study. Patients with history of orthodontic treatment, birth injuries, oral and nasal surgeries, patients who have bone deformities in the craniofacial region, muscular dystrophies, cleft palate, hereditary syndromes like craniofacial dysostosis, etc., were excluded from the study. Selected patients with history of the digit-sucking habit were grouped as the study group and patients without the habit were included in the control group.

\section{Study Design}

It was a cross-sectional study, where the sample size was obtained using the formula:

$$
n=\frac{\left(\mathrm{Z} \alpha^{2}\right)(\mathrm{pq})}{d^{2}}=120
$$

\section{Methods}

Following the inclusion and exclusion criteria, the subjects were selected from the outpatients attending the Department of Pedodontics between January 2017 and August 2018. Total 120 subjects were selected and are equally placed in both the groups (60 in each).

\section{Radiographic Technique}

All radiation protective measures were considered for all the subjects to minimize radiation exposure. The subjects were asked to look straight in a long mirror, which was placed in front of them. Films were obtained from all the subjects by a cephalostat with the Frankfort horizontal plane parallel to the floor and teeth in centric occlusion with relaxed lips Fuji X-ray film $\left(8^{\prime \prime} \times 10^{\prime \prime}\right)$ with speed E were exposed at $80 \mathrm{Kvp}: 40 \mathrm{~mA}$ for 2 seconds from a fixed distance of 60 inches. To obtain a standardized cephalogram, all the radiographs were orientated similarly for all the patients with same amount of magnification.

Further, the radiographs were traced by a single operator on a standard matte acetate tracing paper in a darkened room, with the area of the light box surrounding the cephalogram shielded for optimum landmark identification. Linear and angular measurements that were recorded to assess the dental and skeletal variations have been shown in the Figures 1 to 3 .

\section{Statistical Analysis}

The quantitative analysis of results was performed using the means. The unpaired Student sample's $t$-test was used to compare the mean between digit and nondigit suckers.

\section{Results}

In the present study, the deviations in the dentofacial growth were determined by comparing the lateral cephalogram of digit-sucking with nondigit-sucking children.

On comparing the mean values of skeletal linear variables between the groups, statistical significance was seen for ANS-PNS $(p<0.001)$, CO-GN $(p=0.008)$, N-ANS ( $p=0.007)$, and S-BA ( $p=$ $0.005)$. The significant higher mean values for the variables were noticed with ANS-PNS, CO-GN, and S-BA. Whereas N-ANS elicited reduced mean value in the study group than in controls (Table 1).

Statistical significance was observed for MNI-MNP $(p<0.001)$ and MXI-SNL $(p<0.001)$ when mean dental angular variables were compared (Table 2).

Whereas Table 3 depicts the comparison of mean skeletal angular variables between the groups. It was evident that the mean values for SNA ( $p<0.001)$, SNB ( $p<0.001)$, and MNP-SNL $(P=0.045)$ were significantly higher among the study group as compared to controls.

\section{Discussion}

In the present study, cephalometric linear and angular variables were measured to assess the effects of digit sucking on the maxilla, mandible, and cranial base morphology by measuring incisor angulation, maxillary mandibular lengths, cranial base, and vertical height measurements to evaluate the prognathism, sagittal, and transverse variations in the dentofacial skeleton.

Some significant differences were observed, which can reasonably be attributed to the digit sucking. In the present study, a total of four skeletal linear variables ANS-PNS, CO-GN, S-BA, and $\mathrm{N}$-ANS showed the significant difference between study and control groups. The mean values of ANS-PNS, CO-GN, and S-BA increased significantly in the digit-sucking group $(48.77 \mathrm{~mm}, 43.86 \mathrm{~mm}$, and

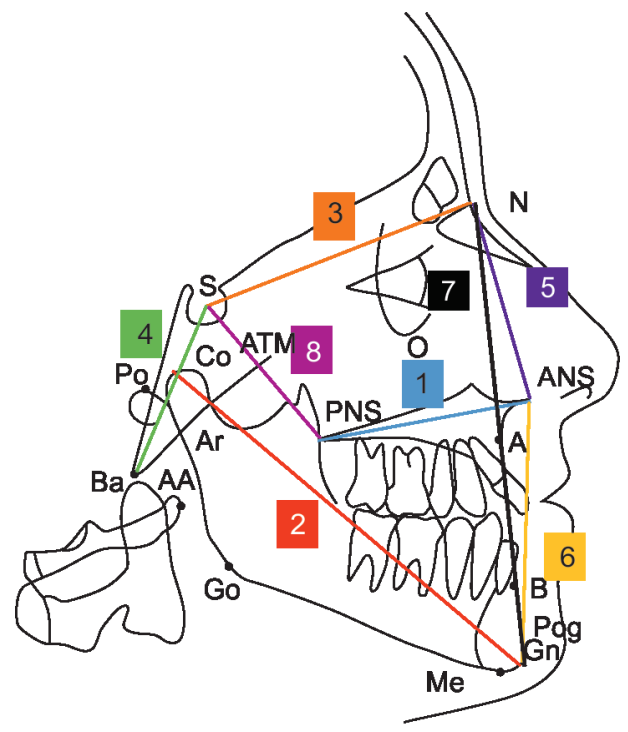

1. Maxillary length (ANS-PNS)

2. Mandibular length (CO-GN)

3. Anterior cranial base length (N-S)

4. Posterior cranial base length (S-BA)

5. Upper anterior facial height (N-ANS)

6. Lower anterior facial height (ANS-GN)

7. Total anterior facial height (N-GN)

8. Posterior facial height (PNS-S)

Fig. 1: Linear variables 


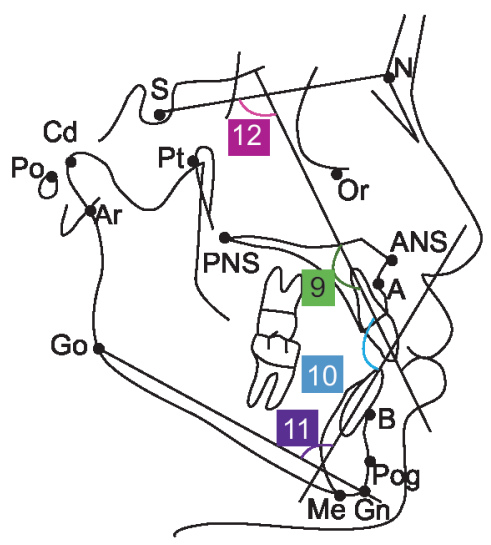

09. Angle between maxillary incisors ANS to PNS (MXI-MXP)

10. Inter incisal angle (MXI-MNI)

11. Angle between mandibular incisors and gonion to gnathion (MNI-MNP)

12. Angle between maxillary incisors and nasion to sella (MXI-SNL)

Fig. 2: Dental angular measurements

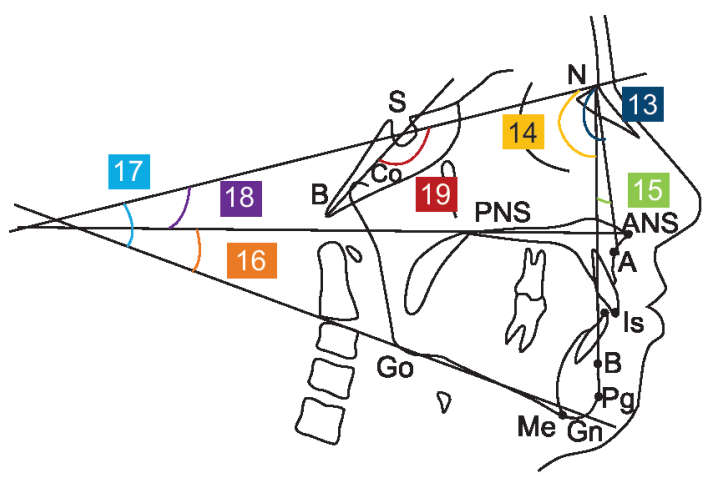

13. Angle between sella nasion to point A (SNA)

14. Angle between sella nasion to point $B$ (SNB)

15. Angle between point A-nasion-point $B$ (ANB)

16. Angle between gonion to gnathion and ANS to PNS (MNP-MXP)

17. Angle between gonion to gnathion and nasion to selle (MNP-SNL)

18. Angle between ANS to PNS and nasion to selle (MXP-SNL)

19. Cranial base angle: Angle between basion selle to nasion (B-S-N)

Fig. 3: Skeletal angular measurements

Table 1: Comparing the mean values of linear variables between the study group and the control group

\begin{tabular}{|c|c|c|c|c|c|}
\hline Linear variables & Study group mean & Control group mean & Difference between means & tvalue & $p$ value \\
\hline ANS-PNS & 48.77 & 44.97 & 3.800 & 5.253 & $0.000^{*}$ \\
\hline CO-GN & 108.63 & 103.47 & 5.167 & 2.691 & $0.008^{*}$ \\
\hline N-ANS & 47.53 & 50.93 & -3.400 & -2.760 & $0.007^{*}$ \\
\hline ANS-GN & 60.17 & 59.60 & 0.567 & 0.375 & 0.709 \\
\hline $\mathrm{N}-\mathrm{GN}$ & 107.47 & 103.67 & 3.800 & 1.536 & 0.127 \\
\hline S-PNS & 44.30 & 44.87 & -0.567 & -0.388 & 0.698 \\
\hline N-S & 70.27 & 69.30 & 0.967 & 0.979 & 0.329 \\
\hline S-BA & 43.86 & 41.20 & 2.664 & 2.881 & $0.005^{*}$ \\
\hline
\end{tabular}

${ }^{*} p$ value $<0.05$ indicates statistically significant. Measurements are expressed in millimeter

Table 2: Comparing the mean values of dental angular variables between the study group and the control group

\begin{tabular}{lccccc}
\hline Dental angular variables & Study group mean & Control group mean & Difference between means & $t$ value & $p$ value \\
\hline MXI-MXP & 116.20 & 118.23 & -2.033 & -0.704 & 0.483 \\
MNI-MNP & 99.17 & 105.33 & -6.167 & -4.122 & $0.000^{*}$ \\
MXI-MNI & 110.80 & 110.23 & 0.567 & 0.305 \\
MXI-SNL & 109.47 & 95.40 & 14.067 & 4.362 & 0.761 \\
\hline
\end{tabular}

${ }^{*} p$ value $<0.05$ indicates statistically significant. Measurements are expressed in millimeter

Table 3: Comparing the mean values of skeletal angular variables between the study group and the control group

\begin{tabular}{|c|c|c|c|c|c|}
\hline Skeletal angular variables & Study group mean & Control group mean & Difference between means & tvalue & $p$ value \\
\hline SNA & 80.50 & 77.37 & 3.133 & 4.755 & $0.000^{*}$ \\
\hline SNB & 76.00 & 73.03 & 2.967 & 4.018 & $0.000^{*}$ \\
\hline ANB & 4.63 & 4.97 & -0.333 & -0.804 & 0.423 \\
\hline BA-S-N & 137.53 & 135.87 & 1.667 & 1.412 & 0.161 \\
\hline MXP-MNP & 24.83 & 25.40 & -0.567 & -0.449 & 0.654 \\
\hline MXP-SNL & 19.50 & 20.57 & -1.067 & 1.219 & 0.225 \\
\hline MNP-SNL & 44.90 & 41.51 & 3.333 & 2.031 & $0.045^{*}$ \\
\hline
\end{tabular}

${ }^{*} p$ value $<0.05$ indicates statistically significant. Measurements are expressed in millimeter

$108.63 \mathrm{~mm}$ ) than those of nondigit suckers $(44.97 \mathrm{~mm}, 41.20 \mathrm{~mm}$, and $103.47 \mathrm{~mm}$ ). This might be due to the digit interference between maxilla and mandible, generating a light long-term continuous force on the maxilla resulting in forward positing of ANS. This habit also causes alterations in the condylar position, creating a gap between the head of the condyle and glenoid fossae, thus stimulating the 
condylar cartilage, which leads to increased measurement from condylon to gnathion. Posterior cranial base length is measured from sella to basion; this has been increased because the altered position of condyle secondarily would have affected the position of basion due to bone remodeling. These results were in agreement with the study done by Taft and Hempstead. ${ }^{6}$

The statistical decrease in the mean of upper anterior facial height (N-ANS) has been noticed in digit suckers $(47.53 \mathrm{~mm})$ than in nondigit suckers $(50.93 \mathrm{~mm})$. This might be due to the resistance offered by the digit to the downward growth of the anterior maxilla tilted the maxillary plane anteriorly upward and posteriorly downward resulting in reduced upper anterior facial height. But these findings are in contrary to the study done by Moore and Mc Donald where they have reported overall rotation of the maxilla without significant reduction in upper anterior facial height. ${ }^{7}$

On intergroup comparison, insignificant differences were noticed in linear skeletal variables when measured from sella to nasion, ANS to gnathion, and nasion to gnathion. These findings are in accordance with the study done by Moore and Mc Donald. ${ }^{7}$

The angle between mandibular incisors and mandibular plane (MNI-MNP) reduced statistically in the study group $\left(99.17^{\circ}\right)$ when compared to the control group $\left(105.33^{\circ}\right)$, depicting accentuated retroclination of mandibular incisors. This might be due to increased perioral muscular activity during digit sucking and this finding was in accordance with the study conducted by Subtelny, ${ }^{8}$ Backlund, ${ }^{9}$ and Gardiner. ${ }^{10}$

Maxillary incisors were significantly proclined relative to the cranial base (MXI-SNL) in the study group $\left(109.47^{\circ}\right)$ when compared to the control group $\left(95.40^{\circ}\right)$; this might be due to the lever effect of the digit creating an anteriorly directed force on the maxillary alveolar process and incisors further resulting in more anteriorly positioned point "A." Similar findings were reported by Moore and Mc Donald ${ }^{7}$ and Willmot. ${ }^{11}$

Generally, increased proclination is characterized by the increased angle between the maxillary plane and maxillary incisors; though the proclination is prominent in most of the digit suckers, the study results did not show a significant increase in this angle. The bidirectional changes such as the increase in the length of maxillary plane and incisors proclination have maintained the angle without much deviation. Hence, the angle between the maxillary plane and maxillary incisors (MXI-MXP) might have not been altered significantly in the study group. The angle between maxillary and mandibular incisors (MXI-MNI) also did not show a significant difference in the study group; this might be due to the increased mandibular length, which led to the bodily movement of mandibular incisors that have maintained the angle.

The mean value of SNA angle in the present study was increased significantly in study group $80.50^{\circ}$ controls $77.37^{\circ}$, indicating maxillary protrusion relative to the cranial base. The reason attributed could be the forces generated during the habit encourage forward growth of the maxilla resulting in more anteriorly positioned point "A." These results are in accordance with the study done by Moore and Mc Donald. ${ }^{7}$

The mean value of SNB angle was significantly increased in the study group $\left(76.00^{\circ}\right)$ than controls $\left(73.00^{\circ}\right)$, indicating mandibular protrusion relative to the cranial base. The reason for this could be that prolonged periods of altered position of the condyle and subsequent bone formation secondary to the habit could have placed the point $B$ in forward position. However, these findings were in contrary to the study results by Moore and Mc Donald. ${ }^{7}$
The angle between gonion to gnathion and nasion to sella (MNP-SNL) suggests growth patterns in individuals; the mean values of this angle were $44.90^{\circ}$ and $41.51^{\circ}$ in study subjects and controls, respectively, indicating a vertical growing facial pattern in digit suckers.

An interesting facet of this study is that along with maxillary changes, mandibular and cranial changes are also evidently emphasizing the deleterious effects of the habit on the growing craniofacial skeleton and it also reflects that growth of the face will differ in both speed and velocity between children with or without the sucking habit, making them more vulnerable to permanent skeletal deviation at a faster rate. To know exactly the habit-related changes, variables such as the position of the digit and the action of the tongue during the sucking process, intensity, duration, and frequency of the habit need to be considered. However, because of the limited samples in the study group, it was not possible to break down into subgroups depending on these variables. Thus opening the paths for further research.

\section{Conclusion}

From the observations of this study, it can be concluded that the changes from the normalcy either dental or skeletal have been limited to maxilla/mandible. The sites far from the oral cavity such as cranial base have not shown significant changes.

Thus evaluation of dental and skeletal changes in a patient with the digit-sucking habit will help to discern the importance of early identification with a reliable diagnostic aid and intercepting them at an early age, thereby ensuring a functional environment adequate for physiological growth that helps in establishing occlusal harmony and dentofacial esthetics.

\section{References}

1. Tulley WJ. Adverse muscle forces - their diagnostic significance. Am J Orthod 1956;42(11):801-814. DOI: 10.1016/0002-9416(56)90088-4.

2. Lear CSC, Catz J, Grossman RC, et al. Measurement of lateral muscle forces on the dental arches. Arch oral Bid 1965;10(4):669-689. DOI: 10.1016/0003-9969(65)90013-0.

3. Sim MJ, Finn SB. Oral habits in children. In: Finn SB. Clinical Pedodontics. 4th ed., Philadelphia: W.B. Saunders Company; 1987. 323-390.

4. Larsson EF, Dahlin KG. The prevalence and the etiology of the initial dummy and finger-sucking habit. Am J Orthod 1985;87(5):432-435. DOI: 10.1016/0002-9416(85)90203-9.

5. Khijmatgar S, Badavannavar A, Saraiya S. Digit sucking as an etiological factor for skeletal discrepancies: a review. Unique J Med Dent Sci 2015;3:63-67.

6. Taft LL, Hempstead NY. A diagnostic study of the dentition, dentofacial pattern and cranial base of prolonged thumb-suckers. Am J Orthod 1966;52(9):703-705. DOI: 10.1016/0002-9416(66)90225-9.

7. Moore MB, Mc Donald JP. A cephalometric evaluation of patients presenting with persistent digit sucking habits. Br J Orthod 1997;24(1):18-23. DOI: 10.1093/ortho/24.1.17.

8. Subtelny JD, Subtelny JD. Oral habits - studies in form, function and therapy. Angle Orthod 1973;43(4):347-383.

9. Backlund E. Facial growth, and the significance of oral habits, mouth breathing and soft tissues for malocclusion. A study of children around the age of 10. Acta Odontol Scand 1963;21(1):9-139. DOI: $10.3109 / 00016356309019775$.

10. Gardiner JH. A survey of malocclusion and some aetiological factors in 1000 sheffield school children. Dental Practitioner 1956;6: 187-198.

11. Willmot DR. Thumb sucking habit and associated dental differences in one of monozygous twins. Br J Orthod 1984;11(4):195-199. DOI: 10.1179/bjo.11.4.195. 